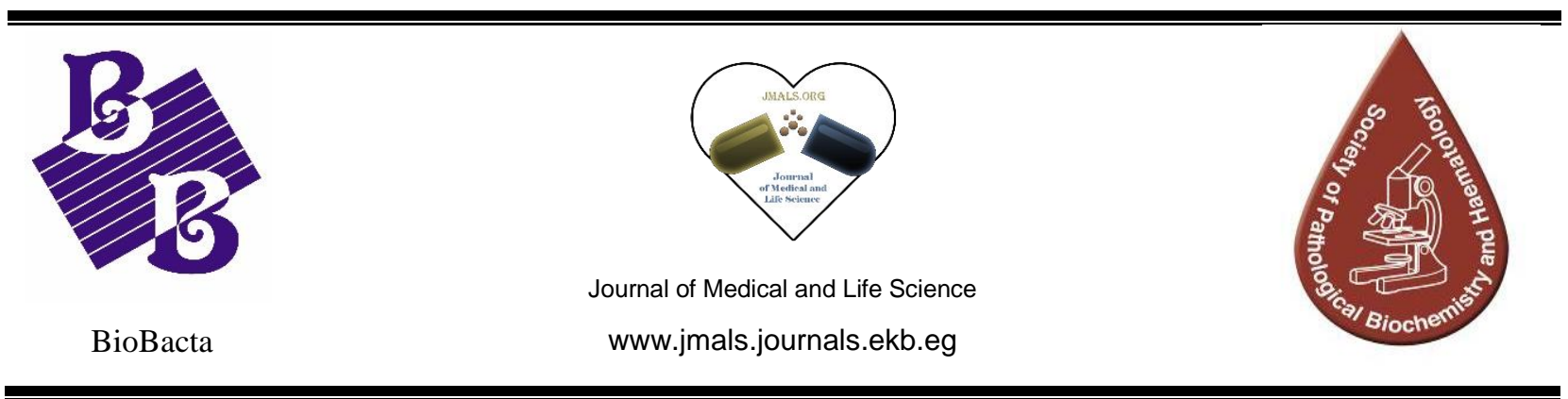

\title{
Efficacy of l-carnitine and silymarin administration on the health-related quality of life in 120 patients with cancer undergoing anthracycline-based chemotherapy
}

\author{
Zeinab Alkasaby Zalat', Mohamed M. Abdel-Latif ${ }^{2}$, Badriyah S. Alotaibi ${ }^{3}$, \\ Mohamed A. Alm El-Din ${ }^{4}$, Neveen A. Kohaf ${ }^{5}$, and Hosny A. Elewa ${ }^{6}$ \\ ${ }^{1}$ Department of Clinical Pharmacy, Faculty of Pharmacy (Girla), Al-Azhar University, Cairo, Egypt. \\ (zeinabalkasaby.pharmg@azhar.edu.eg) \\ ${ }^{2}$ Department of Clinical Pharmacy, Faculty of Pharmacy, Assuit University, Assuit, Egypt. \\ ${ }^{3}$ Department of Pharmaceutical science, Faculty of Pharmacy, Princess of Nourah bint Abdulrahman University, \\ Riyadh, Saudi Arabia \\ ${ }^{4}$ Department of Clinical Oncology, Faculty of Medicine, Tanta University, Tanta, Egypt. \\ ${ }^{5}$ Master degree in pharmaceutical Sciences, Faculty of Pharmacy, Al-Azhar University, Cairo, Egypt. \\ ${ }^{6}$ Department of Pharmacy Practice, Faculty of Pharmacy, Horus University, Dominate City, Egypt. \\ * Corresponding author: helewa@ horus.edu.eg \\ Received May 19, 2020; Accepted June 18, 2020; Published June 21, 2020
}

DOI: 10.21608 /jmals.2020.111233

\begin{abstract}
Objectives: To investigate changes after adding L-carnitine and silymarin compared to anthracycline chemotherapy alone on the health-related quality of life (HRQoL) of women with breast cancer receiving anthracycline-based chemotherapy. Methods: A prospective, randomized comparative, study that included 120 women with breast cancer who received anthracycline in their chemotherapeutic regimen. Patients were divided into three groups, anthracycline alone (control group), chemotherapy and 1-carnitine (1-carnitine group), and chemotherapy plus silymarin (silymarin group). HRQoL was evaluated using the EORTC QLQ-C30 and EORTC QLQ-BR23 instruments 7 days before chemotherapy and after the third month of chemotherapy. Results: On the application of (EORTC QLQ-C30), there was a significant decrease in global health status/QoL score, functional scale scores from baseline to after three months $(P \leq 0.001)$ within the control group and a significant increase in symptom scale scores from baseline to after three months. In l-carnitine and silymarin groups, there was a nonsignificant difference in the scale scores. On the application of (EORTC QLQ-BR23), there was a significant decrease in functional scale scores $(\mathrm{p} \leq 0.001)$ within the control group and a significant increase in symptom scale scores $(\mathrm{p} \geq 0.05)$. In the 1-carnitine and silymarin groups, there was a non-significant difference in functional scale scores from baseline to after three months ( $\mathrm{p} \geq 0.05$ ). Conclusions: QOL was negatively affected by chemotherapy. For BC cases, HRQoL becomes typically worse during the third month of chemotherapy compared with the pretreatment duration. The addition of 1-carnitine and silymarin to anthracycline-based chemotherapy showed improvement in the health-related quality of life of cancer patients.
\end{abstract}

Keywords: Anthracycline, silymarin, 1-carnitine, health-related quality of life, breast cancer. 


\section{Introduction}

The distribution of cancer is currently growing, and the number of new patients is set to grow from 14 million in 2012 to 22 million annually by 2030 [1]. The second most common disease in the world in new cases (1.7 million cases) is breast cancer (BC), and the fifth category is regarded as a cause of death. [2]. With survival rates rising during $\mathrm{BC}$ therapy, greater consideration is given to improving health-related quality of life (HRQoL) during and following cancer drugs. Although anthracycline chemotherapy is associated with positive benefits in decreasing the risk of $\mathrm{BC}$ recurrence [3], it also affects negatively the HRQoL of survivors [4]. Furthermore, the reality that $\mathrm{BC}$ is gradually identified in earlier stages as a result of screening initiatives further increases the number of people who have obtained curative-intent adjuvant chemotherapy. The symptomatology and adverse effects profile of anthracycline chemotherapy on HRQoL should be taken into consideration. For example, chemotherapy induces important effects in BC people, such as exhaustion, febrile neutropenia, depression, dyspnea, discomfort, nausea, and vomiting. [5]. In addition, emotional distress, such as confusion or concern of recurrence and posttraumatic stress, pain, and work strain can include carcinogenic sequelae [6].

In the framework of culture and value systems, the idea of the quality of life (QoL) can be described as a sense of identity in its place in life and in relation to its objectives, desires, values, and concerns [7]. When this term is just linked to health expectations, the expression is titled HRQoL. This expression is a multi-domain term, representing the patient's general perception of the impact of illness and treatment on other aspects of life. [8]. The expression HRQoL is therefore used to include those elements that are usually not addressed in health contexts (such as income independence and environmental quality). Focusing on the development of HRQoL thus requires analyzing nearly all healthrelated aspects of life [9].

In order to improve HRQoL and maintain emotional, social, and physical wellness, it is thus essential to understand the requirements of patients, besides the control of clear signs and symptoms throughout the therapy. Therefore, it is important to search for new strategies to improve HRQoL in cancer patients, such as adding 1-carnitine and silymarin to patient's treatment protocols.

L-carnitine is important for the synthesis of longchain free fatty acids into acylcarnitines and their subsequent transfer to the mitochondrial matrix where they are beta-oxidized in the production of cellular resources. L-carnitine's exogenous supplemental therapies seem to support anorexia, chronic fatigue, cardiovascular disease, diphtheria, hypoglycemia, male infertility, and muscular illness [10].

Silymarin is a nontoxic natural polyphenolic flavonoid extracted from the seeds of the plant milk thistle (Silybum marianum), which is an ancient medicinal plant for the treatment of various liver diseases [11]. Due to its strong antioxidant and tissue regenerative properties, silymarin is being studied as a hepatic, neural, renal, and cardiac protective ingredient. [12]. Silymarin could be helpful in patients with oncology, particularly, for reducing the side effects of cancer chemotherapy [10]. In common cancers such as lung prostatic, stomach, breast, bladder, and hepatocellular carcinoma, even, silymarin has an anticancer effect [13].

This research aimed to measure QOL in BC patients and compare the algorithms before and after chemotherapy and determine the impact of l-carnitine and silymarin on HRQoL.

\section{Method}


A prospective study to assess HRQoL in 120 Egyptian BC patients who performed the first oncological consultation at the Oncology Department, Tanta University Hospital, Egypt. Women $\geq 18$ years of age, histologically documented $\mathrm{BC}$, which has an intervention with breast surgery, and which depends on adjuvant or neoadjuvant chemotherapy as anthracycline, are included.

Patients were divided into three groups: control group (anthracycline- based regimen alone, $\mathrm{n}=40$ ), 1-carnitine group (anthracycline-based regimen +1 -carnitine $1 \mathrm{~g}$ daily, $n=40$ ) and silymarin group (anthracycline- based regimen + silymarin $140 \mathrm{mg}$ daily, $\mathrm{n}=40$ )

The evaluation of the patient's HRQoL was evaluated using the EORTC QLQ-C30 (European Organisation for Research and Treatment of Cancer Quality of Life Core Questionnaire) and EORTC QLQ-BR23 (EORTC BC-specific Quality of Life Questionnaire). The patients performed questionnaires in two stages (i. e., one week before the beginning of the chemotherapy (baseline) and in the third month of therapy, roughly in the 4th period (3-month follow-up), all of these instruments are checked, converted into Arabic, formal, and self-administrative information from medical records such as sociodemographic details (educational level and marital condition), menopause status, family history of cancers, and data were obtained from the Eastern Cooperative Oncology Group scale, measured no more than two weeks before research enrollment. The questionnaire was evaluated according to the systematic approach proposed by the EORTC Group. The research was formulated and performed in compliance with the ethical values of the Standards for Good Clinical Practice and the Helsinki Declaration. The methodology of research was accepted by the
National Research Ethics Committee at Tanta University with acceptance number (32551/09/2018), and written informed consent of all patients was received.

\section{Drugs:}

L-carnitine ${ }^{\circledR} 500 \mathrm{mg}$ capsules obtained from (MEPACO)

Silymarin (Legalon ® $140 \mathrm{mg}$ capsule obtained from (MEDA).

One-way analysis of variance test (one-way ANOVA) followed by LSD post hoc test was used to assess any significant differences among the three groups. A paired t-test was used to assess any significant differences within each group at baseline and after chemotherapy. All probability values presented were two-tailed, and $p \leq 0.05$ was considered statistically significant.

\section{Results}

1- Study population

Results concerning sociodemographic and clinical characteristics from the 120 patients included in the study are described in Tables 1, 2, and 3.

There were no significant differences in age, marital status, education level, and menopausal state between the studied groups ( $p$-value $\geq 0.05$ ) as shown in Table 1.

In Tables 2 and 3, there were also non-significant differences regarding family history of cancer (Eastern Co-Operative Oncology Group) ECOG performance, a model of breast cancer detection, and stage of breast cancer between the studied groups ( $\mathrm{p}$-value $\geq 0.05$ ) 
Table (1): Demographic data of the studied groups

\begin{tabular}{|c|c|c|c|c|}
\hline \multirow[b]{2}{*}{ Variable } & \multicolumn{3}{|l|}{ Groups } & \multirow[b]{2}{*}{$\begin{array}{l}P \text { - } \\
\text { value }\end{array}$} \\
\hline & $\begin{array}{l}\text { Control Group } \\
\mathrm{N}=\mathbf{4 0}\end{array}$ & $\begin{array}{l}\text { l-carnitine group } \\
\qquad \mathrm{N}=40\end{array}$ & $\begin{array}{l}\text { Silymarin group } \\
\qquad \mathrm{N}=\mathbf{4 0}\end{array}$ & \\
\hline Age (years) Mean \pm SD & $44.455 \pm 9.47$ & $45.64 \pm 9.941$ & $44.68 \pm 12.44$ & 0.61 \\
\hline $\begin{array}{l}\text { Marital status } \\
\text { Married } \\
\text { Single }\end{array}$ & $\begin{array}{l}43(71.6 \%) \\
17(28.3 \%)\end{array}$ & $\begin{array}{l}18(72 \%) \\
7(28 \%)\end{array}$ & $\begin{array}{l}19(76 \%) \\
6(24 \%)\end{array}$ & 0.775 \\
\hline $\begin{array}{l}\text { Education level } \\
\text { Elementary or middle school } \\
\text { High school } \\
\text { College }\end{array}$ & $\begin{array}{l}55(91.6 \%) \\
3(5 \%) \\
2(3.33 \%)\end{array}$ & $\begin{array}{l}21(84 \%) \\
3(12 \%) \\
1(4 \%)\end{array}$ & $\begin{array}{l}22(88 \%) \\
2(8 \%) \\
1(4 \%)\end{array}$ & 0.726 \\
\hline $\begin{array}{l}\text { Menopausal state } \\
\text { Premenopausal } \\
\text { Perimenopausal } \\
\text { Postmenopausal }\end{array}$ & $\begin{array}{l}10(16.6 \%) \\
33(55 \%) \\
17(28.3 \%)\end{array}$ & $\begin{array}{l}3(12 \%) \\
18(72 \%) \\
4(16 \%)\end{array}$ & $\begin{array}{l}4(16 \%) \\
17(68 \%) \\
4(16 \%)\end{array}$ & 0.700 \\
\hline
\end{tabular}

Age represented in mean \pm standard deviation

Data are represented as number and percentage. $p \leq 0.05$ value considered significant

Table (2): Demographic data of the studied groups

\begin{tabular}{|c|c|c|c|c|}
\hline \multirow[b]{2}{*}{ Variable } & \multicolumn{3}{|c|}{ Groups } & \multirow[b]{2}{*}{$P$ value } \\
\hline & $\begin{array}{l}\text { Control } \\
\text { Group } \\
\mathrm{N}=\mathbf{4 0}\end{array}$ & $\begin{array}{l}\text { l-carnitine group } \\
\mathrm{N}=40\end{array}$ & $\begin{array}{l}\text { Silymarin group } \\
\mathrm{N}=\mathbf{4 0}\end{array}$ & \\
\hline $\begin{array}{l}\text { Family history of cancer } \\
\text { No } \\
\text { Yes (breast) } \\
\text { Yes (breast and other) } \\
\text { Yes (other) }\end{array}$ & $\begin{array}{l}25(41.6 \%) \\
20(33.3 \%) \\
5(8.3 \%) \\
10(16.6 \%)\end{array}$ & $\begin{array}{l}10(40 \%) \\
5(20 \%) \\
6(24 \%) \\
4(16 \%)\end{array}$ & $\begin{array}{l}11(44 \%) \\
6(24 \%) \\
5(20 \%) \\
3(12 \%)\end{array}$ & 0.815 \\
\hline $\begin{array}{l}\text { ECOG performance status } \\
0 \\
1\end{array}$ & $\begin{array}{l}55(91.6 \%) \\
5(8.33 \%)\end{array}$ & $\begin{array}{l}23(92 \%) \\
2(8 \%)\end{array}$ & $\begin{array}{l}22(88 \%) \\
3(12 \%)\end{array}$ & 0.850 \\
\hline $\begin{array}{l}\text { Model of breast cancer detection } \\
\text { Screen detected } \\
\text { Symptomatic } \\
\text { Unknown }\end{array}$ & $\begin{array}{l}30(50 \%) \\
20(33.3 \%) \\
10(16.6 \%)\end{array}$ & $\begin{array}{l}16(64 \%) \\
5(20 \%) \\
4(16 \%)\end{array}$ & $\begin{array}{l}15(60 \%) \\
6(24 \%) \\
4(16 \%)\end{array}$ & 0.674 \\
\hline
\end{tabular}

ECOG: Eastern Cooperative Oncology Group 
Table (3): Distribution of patients regarding pathology and stage in the studied groups

\begin{tabular}{|c|c|c|c|c|c|c|c|}
\hline \multirow[t]{3}{*}{ Variable } & \multicolumn{6}{|c|}{ Groups } & \multirow{3}{*}{$P$-Value } \\
\hline & \multicolumn{2}{|c|}{ Control } & \multicolumn{2}{|c|}{ L- Carnitine } & \multicolumn{2}{|c|}{ Silymarin } & \\
\hline & $\mathrm{N}$ & $\%$ & $\mathrm{~N}$ & $\%$ & $\mathrm{~N}$ & $\%$ & \\
\hline Stage: & & & & & & & \\
\hline Breast adjuvant & 27 & $(67.5 \%)$ & 33 & $(82.5 \%)$ & 31 & $(77.5 \%)$ & \\
\hline Breast & 4 & $(10 \%)$ & 2 & $(5 \%)$ & 6 & $(15 \%)$ & 0.742 \\
\hline neoadjuvant & 4 & $(10 \%)$ & 1 & $(2.5 \%)$ & 2 & $(5 \%)$ & \\
\hline $\begin{array}{l}\text { Breast } \\
\text { metastatic }\end{array}$ & 5 & $(12.5 \%)$ & 4 & $(10 \%)$ & 1 & $(2.5 \%)$ & \\
\hline
\end{tabular}

Data are represented as number and percentage. $p \leq 0.05$ value considered significant

Age represented in mean \pm standard deviation

$\mathrm{p} \leq 0.05$ value considered significant

\section{2-HRQoL scores}

Application of (EORTC QLQ-C30). There was a significant decrease in global health status/QoL score, physical functioning, role functioning, emotional functioning, and social functioning scores from baseline to after three months $(p \leq 0.001)$ within the control group. There was a significant increase in symptoms scale includefatigue, nausea and vomiting, dyspnea, insomnia, appetite loss, constipation, and diarrhea scores from baseline to after three months. Table (4) and figures 1-3

In the 1-carnitine group, there was a nonsignificant difference in global health status/QoL score, physical functioning, role functioning, emotional functioning, and social functioning scores from baseline to after three months $(p \geq 0.05)$. There was also a non-significant change in symptoms scale include pain, insomnia, appetite loss, and constipation scores from baseline to after three months. Table (4) and figures 1-3
In the silymarin group, there was a non-significant difference in global health status/QoL score, physical functioning, role functioning, emotional functioning, and social functioning scores from baseline to after three months $(\mathrm{p} \geq 0.05)$ and there was also a non-significant change in symptom scale include fatigue, pain, insomnia, appetite loss, and constipation scores from baseline to after three months. Table (4) and figures 1-3

\section{Application of (EORTC QLQ-BR23).}

There was a significant decrease in functional scale include body image score and a significant decrease in sexual functioning, sexual enjoyment scores from baseline to after three months $(p \leq 0.001)$ within the control group, and there was a significant increase in symptoms scale include systemic therapy side effects and breast symptom scores from baseline to after three months $(\mathrm{p} \geq 0.05)$. Table (5) and figures 4,5

In the 1-carnitine group, there was a non-significant difference in functional scale include body image 
score, sexual functioning, and sexual enjoyment scores from baseline to after three months $(\mathrm{p} \geq 0.05)$. Table (5) and figures 4,5
In the silymarin group, there was a non-significant difference in functional scale include body image score, sexual functioning, sexual enjoyment, and arm symptom scores from baseline to after three months. Table (5) and figures 4,5

Table 4. Comparison of HRQoL between the study segments (EORTC QLQ-C30).

\begin{tabular}{|c|c|c|c|c|c|c|}
\hline \multirow{3}{*}{ Variable } & \multicolumn{6}{|c|}{ Groups } \\
\hline & \multicolumn{2}{|c|}{$\begin{array}{l}\text { Control Group } \\
\mathrm{N}=40\end{array}$} & \multicolumn{2}{|c|}{$\begin{array}{l}\text { l-carnitine Group } \\
\mathrm{N}=40\end{array}$} & \multicolumn{2}{|c|}{$\begin{array}{l}\text { Silymarin Group } \\
\mathrm{N}=40\end{array}$} \\
\hline & At base line & After 3 months & At base line & After 3 months & At base line & After 3 months \\
\hline \multicolumn{7}{|c|}{ Global health status/ QoL } \\
\hline $\begin{array}{l}\text { Global } \\
\text { health } \\
\text { status/ QoL }\end{array}$ & $77.6 \pm 16.02$ & $55.18 \pm 15.3$ & $76.7 \pm 15.6$ & $71.2 \pm 13.2$ & $78.7 \pm 13.5$ & $77.4 \pm 14.6$ \\
\hline$P$-value & \multicolumn{2}{|c|}{$0.001^{*}$} & \multicolumn{2}{|c|}{0.1847} & \multicolumn{2}{|c|}{0.745} \\
\hline \multicolumn{7}{|c|}{ functional scales } \\
\hline $\begin{array}{l}\text { Physical } \\
\text { functioning }\end{array}$ & $89.73 \pm 4.51$ & $67.5 \pm 10.33$ & $90.1 \pm 4.88$ & $88.3 \pm 4.093$ & $88.6 \pm 2.89$ & $87.8 \pm 11.3$ \\
\hline$P$-value & \multicolumn{2}{|l|}{$0.001 *$} & \multicolumn{2}{|c|}{0.1641} & \multicolumn{2}{|c|}{0.7331} \\
\hline $\begin{array}{l}\text { Role } \\
\text { functioning }\end{array}$ & $80.78 \pm 19.6$ & $52.3 \pm 18.3$ & $79.3 \pm 15.4$ & $73.1 \pm 14.6$ & $80.4 \pm 16.8$ & $79.6 \pm 14.5$ \\
\hline$P$-value & \multicolumn{2}{|c|}{$0.001 *$} & \multicolumn{2}{|c|}{0.1506} & \multicolumn{2}{|c|}{0.8577} \\
\hline $\begin{array}{l}\text { Emotional } \\
\text { functioning }\end{array}$ & $72.3 \pm 19.3$ & $48.32 \pm 20.5$ & $70.8 \pm 17.5$ & $58.3 \pm 25.6$ & $71.6 \pm 18.7$ & $68.4 \pm 23.4$ \\
\hline$P$-value & \multicolumn{2}{|c|}{$0.001 *$} & \multicolumn{2}{|l|}{$0.0495^{*}$} & \multicolumn{2}{|l|}{0.595} \\
\hline $\begin{array}{l}\text { Cognitive } \\
\text { functioning }\end{array}$ & $83.4 \pm 20.2$ & $75.6 \pm 19.8$ & $85.1 \pm 21.3$ & $83.6 \pm 16.8$ & $80.6 \pm 17.5$ & $80.3 \pm 20.6$ \\
\hline$P$-value & \multicolumn{2}{|l|}{0.1744} & \multicolumn{2}{|l|}{0.783} & \multicolumn{2}{|l|}{0.956} \\
\hline $\begin{array}{l}\text { Social } \\
\text { functioning }\end{array}$ & $87.3 \pm 15.4$ & $59.6 \pm 20.14$ & $88.6 \pm 19.5$ & $89.3 \pm 18.6$ & $86.7 \pm 17.5$ & $88.4 \pm 21.4$ \\
\hline$P$-value & \multicolumn{2}{|l|}{0.001} & \multicolumn{2}{|l|}{0.897} & \multicolumn{2}{|l|}{0.759} \\
\hline \multicolumn{7}{|c|}{ Symptom scales } \\
\hline Fatigue & $14.8 \pm 2.3$ & $58.7 \pm 14.3$ & $13.6 \pm 5.3$ & $18.6 \pm 1.3$ & $15.3 \pm 2.2$ & $16.4 \pm 1.3$ \\
\hline$P$-value & \multicolumn{2}{|l|}{0.001} & \multicolumn{2}{|l|}{0.001} & \multicolumn{2}{|l|}{0.0346} \\
\hline $\begin{array}{l}\text { Nausea and } \\
\text { vomiting }\end{array}$ & $1.02 \pm 0.02$ & $12.6 \pm 1.5$ & $1.5 \pm 1.1$ & $5.6 \pm 2.1$ & $1.8 \pm 1.2$ & $4.7 \pm 3.2$ \\
\hline$P$-value & \multicolumn{2}{|l|}{0.001} & \multicolumn{2}{|l|}{0.001} & \multicolumn{2}{|l|}{0.001} \\
\hline
\end{tabular}




\begin{tabular}{|c|c|c|c|c|c|c|}
\hline \multirow{3}{*}{ Variable } & \multicolumn{6}{|c|}{ Groups } \\
\hline & \multicolumn{2}{|c|}{$\begin{array}{l}\text { Control Group } \\
\mathrm{N}=\mathbf{4 0}\end{array}$} & \multicolumn{2}{|c|}{$\begin{array}{l}\text { l-carnitine Group } \\
\mathrm{N}=\mathbf{4 0}\end{array}$} & \multicolumn{2}{|c|}{$\begin{array}{l}\text { Silymarin Group } \\
\mathrm{N}=\mathbf{4 0}\end{array}$} \\
\hline & At base line & After 3 months & At base line & After 3 months & At base line & After 3 months \\
\hline Pain & $28.3 \pm 23.2$ & $36.3 \pm 24.2$ & $27.3 \pm 12.5$ & $30.5 \pm 21.2$ & $29.1 \pm 15.6$ & $31.2 \pm 18.3$ \\
\hline$P$-value & \multicolumn{2}{|l|}{0.239} & \multicolumn{2}{|l|}{0.518} & \multicolumn{2}{|l|}{0.664} \\
\hline Dyspnoea & $7.6 \pm 4.3$ & $18.2 \pm 5.7$ & $7.9 \pm 2.6$ & $15.2 \pm 1.8$ & $7.5 \pm 2.8$ & $10.6 \pm 3.5$ \\
\hline$P$-value & \multicolumn{2}{|l|}{0.001} & \multicolumn{2}{|l|}{0.001} & \multicolumn{2}{|l|}{0.0011} \\
\hline Insomnia & $28.3 \pm 19.3$ & $52.6 \pm 23.2$ & $29.2 \pm 12.5$ & $33.5 \pm 18.6$ & $28.7 \pm 21.2$ & $32.4 \pm 18.7$ \\
\hline$P$-value & \multicolumn{2}{|l|}{0.001} & \multicolumn{2}{|l|}{0.001} & \multicolumn{2}{|l|}{0.452} \\
\hline $\begin{array}{l}\text { Appetite } \\
\text { loss }\end{array}$ & $7.4 \pm 3.2$ & $22.3 \pm 14.3$ & $6.5 \pm 2.8$ & $8.6 \pm 5.2$ & $7.6 \pm 1.5$ & $6.6 \pm 12.5$ \\
\hline$P$-value & \multicolumn{2}{|l|}{0.001} & \multicolumn{2}{|l|}{0.0818} & \multicolumn{2}{|l|}{0.0928} \\
\hline Constipation & $18.6 \pm 14.5$ & $33.5 \pm 17.6$ & $17.5 \pm 12.2$ & $20.3 \pm 14.8$ & $19.3 \pm 14.3$ & $17.2 \pm 12.3$ \\
\hline$P$-value & \multicolumn{2}{|l|}{0.002} & \multicolumn{2}{|l|}{0.469} & \multicolumn{2}{|l|}{0.5803} \\
\hline Diarrhoea & $1.02 \pm 2.2$ & $9.3 \pm 4.9$ & $1.3 \pm 2.1$ & $6.2 \pm 5.3$ & $1.5 \pm 2.1$ & $3.5 \pm 2.5$ \\
\hline$P$-value & \multicolumn{2}{|l|}{0.001} & \multicolumn{2}{|l|}{0.001} & \multicolumn{2}{|l|}{0.0036} \\
\hline $\begin{array}{l}\text { Financial } \\
\text { difficulties }\end{array}$ & $27.3 \pm 26.3$ & $31.2 \pm 21.3$ & $26.3 \pm 19.8$ & $30.2 \pm 27.3$ & $25.6 \pm 21.3$ & $32.1 \pm 28.3$ \\
\hline$P$-value & \multicolumn{2}{|l|}{0.567} & \multicolumn{2}{|l|}{0.568} & \multicolumn{2}{|l|}{0.363} \\
\hline
\end{tabular}

Table 5. Comparison of HRQoL between study segments. Specific questionnaire for BC (EORTC QLQ-BR23).

\begin{tabular}{|c|c|c|c|c|c|c|}
\hline \multirow{3}{*}{ Variable } & \multicolumn{6}{|c|}{ Groups } \\
\hline & \multicolumn{2}{|c|}{$\begin{array}{l}\text { Control Group } \\
\mathrm{N}=\mathbf{4 0}\end{array}$} & \multicolumn{2}{|c|}{$\begin{array}{l}\text { l-carnitine Group } \\
\mathrm{N}=\mathbf{4 0}\end{array}$} & \multicolumn{2}{|c|}{$\begin{array}{l}\text { Silymarin Group } \\
\mathrm{N}=40\end{array}$} \\
\hline & At base line & After 3 months & At base line & After 3 months & At base line & After 3 months \\
\hline \multicolumn{7}{|c|}{ Functional scales } \\
\hline Body image & $90.66 \pm 8.51$ & $65.5 \pm 12.33$ & $90.1 \pm 8.88$ & $88.3 \pm 8.093$ & $87.6 \pm 7.89$ & $88.8 \pm 18.3$ \\
\hline$P$-value & \multicolumn{2}{|l|}{$0.001 *$} & \multicolumn{2}{|c|}{0.4575} & \multicolumn{2}{|c|}{0.7647} \\
\hline $\begin{array}{l}\text { Sexual } \\
\text { functioning }\end{array}$ & $52.78 \pm 19.6$ & $16.3 \pm 15.3$ & $52.3 \pm 16.4$ & $44.1 \pm 15.6$ & $51.3 \pm 14.8$ & $48.3 \pm 19.5$ \\
\hline$P$-value & \multicolumn{2}{|c|}{$0.001 *$} & \multicolumn{2}{|l|}{0.0763} & \multicolumn{2}{|c|}{0.542} \\
\hline $\begin{array}{l}\text { Sexual } \\
\text { enjoyment } \#\end{array}$ & $69.3 \pm 19.7$ & $28.32 \pm 21.5$ & $70.7 \pm 17.5$ & $33.3 \pm 25.6$ & $71.7 \pm 15.7$ & $66.4 \pm 13.4$ \\
\hline$P$-value & \multicolumn{2}{|c|}{$0.001 *$} & \multicolumn{2}{|l|}{$0.001 *$} & \multicolumn{2}{|l|}{0.02054} \\
\hline
\end{tabular}




\begin{tabular}{|c|c|c|c|c|c|c|}
\hline \multirow{3}{*}{ Variable } & \multicolumn{6}{|c|}{ Groups } \\
\hline & \multicolumn{2}{|c|}{$\begin{array}{l}\text { Control Group } \\
\mathrm{N}=\mathbf{4 0}\end{array}$} & \multicolumn{2}{|c|}{$\begin{array}{l}\text { l-carnitine Group } \\
\mathrm{N}=\mathbf{4 0}\end{array}$} & \multicolumn{2}{|c|}{$\begin{array}{l}\text { Silymarin Group } \\
\mathrm{N}=\mathbf{4 0}\end{array}$} \\
\hline & At base line & After 3 months & At base line & After 3 months & At base line & After 3 months \\
\hline $\begin{array}{l}\text { Future } \\
\text { perspective }\end{array}$ & $33.4 \pm 20.2$ & $39.6 \pm 19.8$ & $34.1 \pm 21.3$ & $35.2 \pm 16.8$ & $32.6 \pm 17.5$ & $33.3 \pm 20.6$ \\
\hline$P$-value & \multicolumn{2}{|l|}{0.278} & \multicolumn{2}{|l|}{0.8402} & \multicolumn{2}{|l|}{0.8975} \\
\hline \multicolumn{7}{|c|}{ Symptom scales } \\
\hline $\begin{array}{l}\text { Systemic } \\
\text { therapy side } \\
\text { effects }\end{array}$ & $8.8 \pm 9.3$ & $58.7 \pm 18.3$ & $7.6 \pm 6.3$ & $48.6 \pm 16.3$ & $8.3 \pm 7.2$ & $36.4 \pm 20.3$ \\
\hline$P$-value & \multicolumn{2}{|l|}{0.001} & \multicolumn{2}{|l|}{0.001} & \multicolumn{2}{|l|}{0.001} \\
\hline $\begin{array}{l}\text { Breast } \\
\text { symptoms }\end{array}$ & $32.3 \pm 23.2$ & $18.6 \pm 21.5$ & $33.5 \pm 21.1$ & $17.6 \pm 22.1$ & $32.8 \pm 21.2$ & $19.3 \pm 23.2$ \\
\hline$P$-value & \multicolumn{2}{|l|}{0.035} & \multicolumn{2}{|l|}{0.0123} & \multicolumn{2}{|l|}{0.0406} \\
\hline $\begin{array}{l}\text { Arm } \\
\text { symptoms }\end{array}$ & $34.3 \pm 23.2$ & $20.3 \pm 24.2$ & $35.3 \pm 12.5$ & $22.5 \pm 21.2$ & $33.1 \pm 15.6$ & $25.2 \pm 18.3$ \\
\hline$P$-value & \multicolumn{2}{|l|}{0.0421} & \multicolumn{2}{|l|}{0.0123} & \multicolumn{2}{|l|}{0.107} \\
\hline $\begin{array}{l}\text { Upset by } \\
\text { hair loss }\end{array}$ & NA & $68.2 \pm 35.7$ & NA & $65.2 \pm 31.8$ & NA & $62.6 \pm 35.5$ \\
\hline$P$-value & & & & & & \\
\hline
\end{tabular}

*Statistically significant $p$-value from the Wilcoxon test.

NA, not applicable; there was no valid information available. \# According to the EORTC Scoring Manual [14], the variation in the number of responses in EORTC QLQ-BR23 is predicted since the fields 'sexual enjoyment' and 'upset by hair loss' do not apply when the responses related to these scales are 'no'.

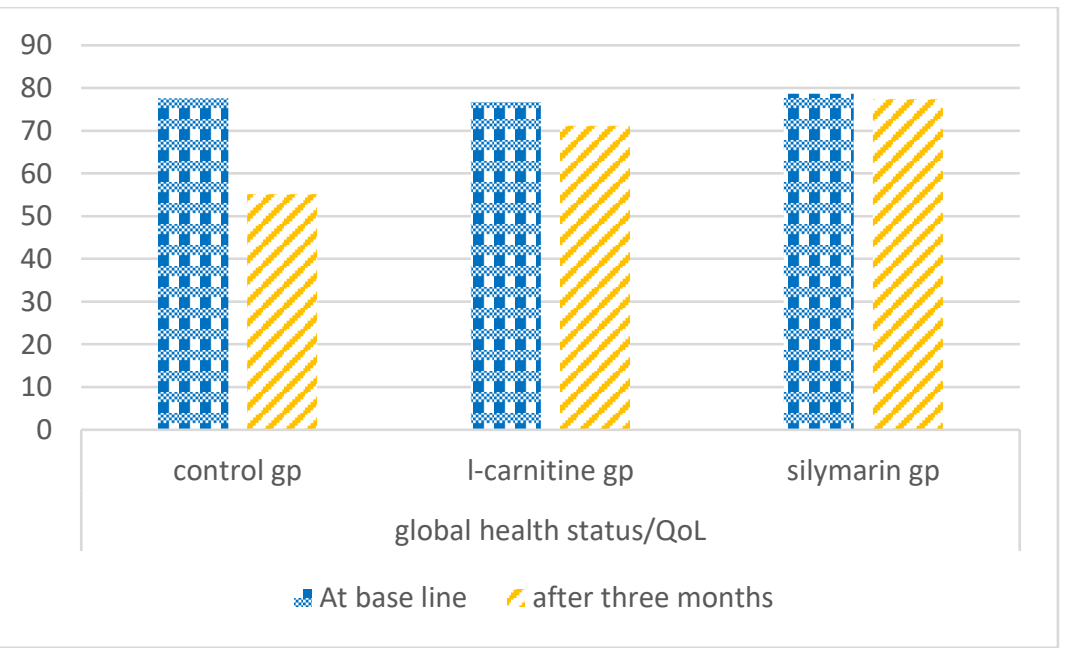

Figure (1): Comparison between studied groups regarding global health status (EORTC QLQ-C30) 

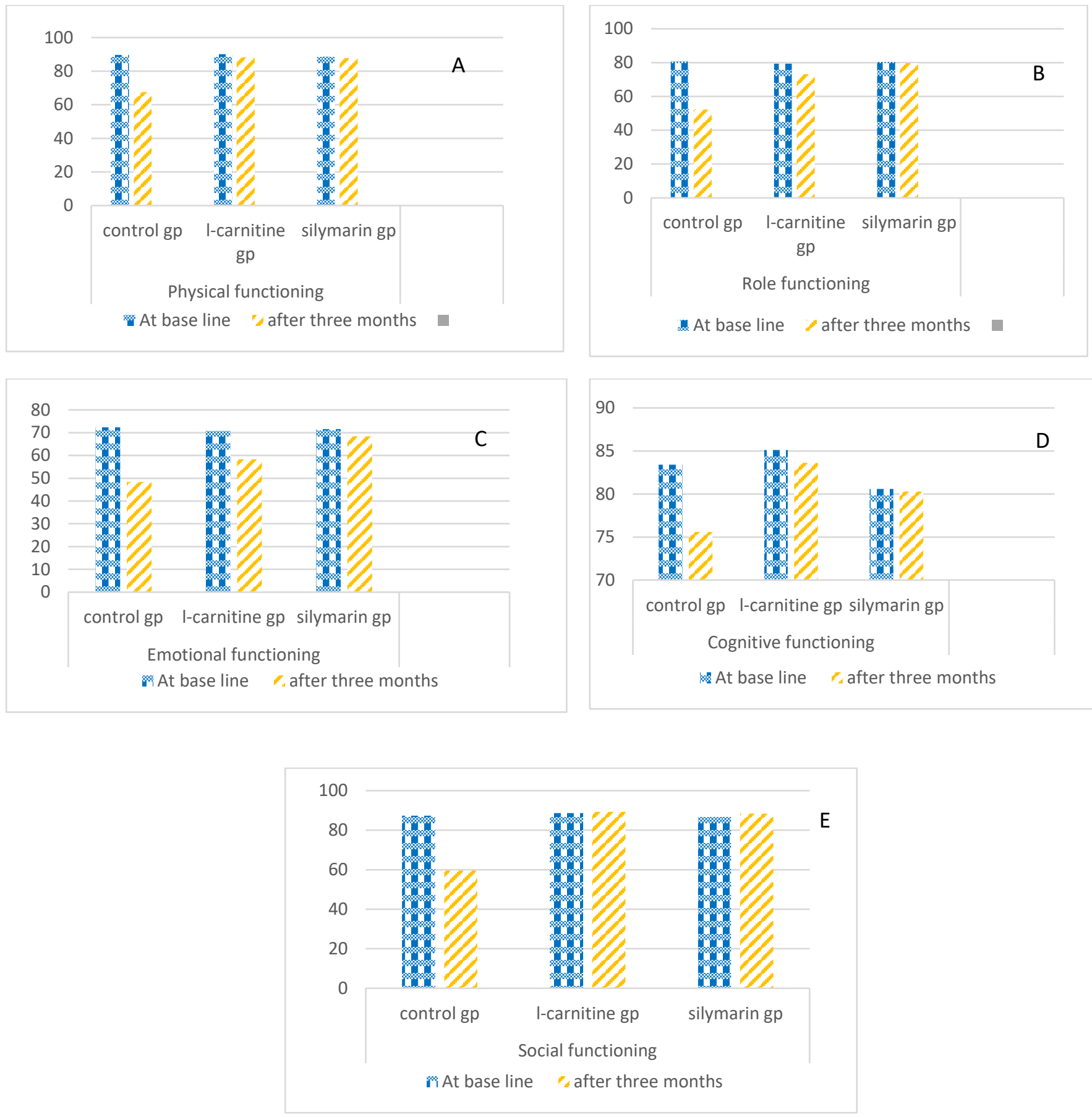

Figure (2): Comparison between studied groups regarding functional scale: A: physical functioning, B: role functioning, C: emotional functioning, D: cognitive functioning, E: social functioning (EORTC QLQ-C30) 

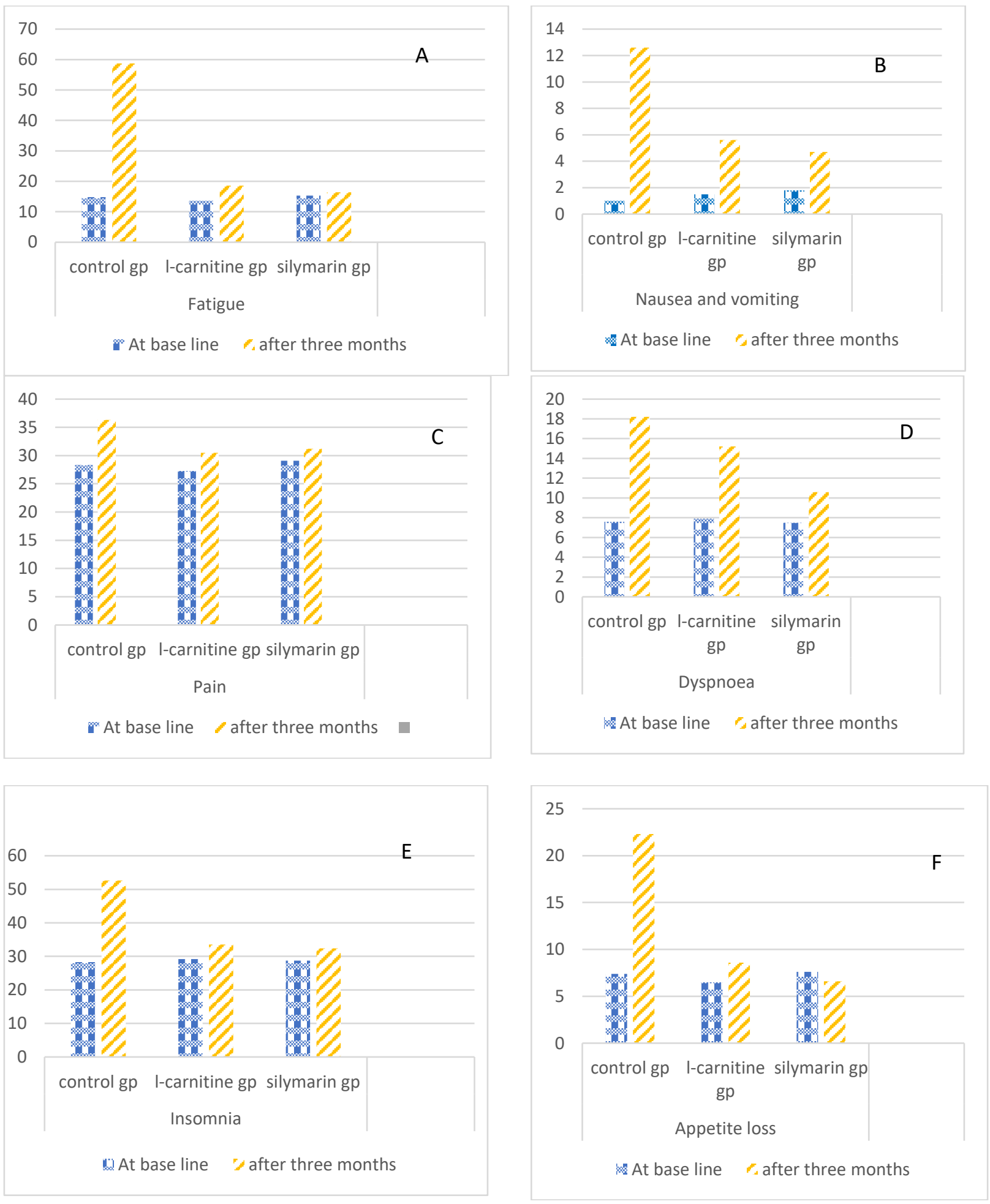

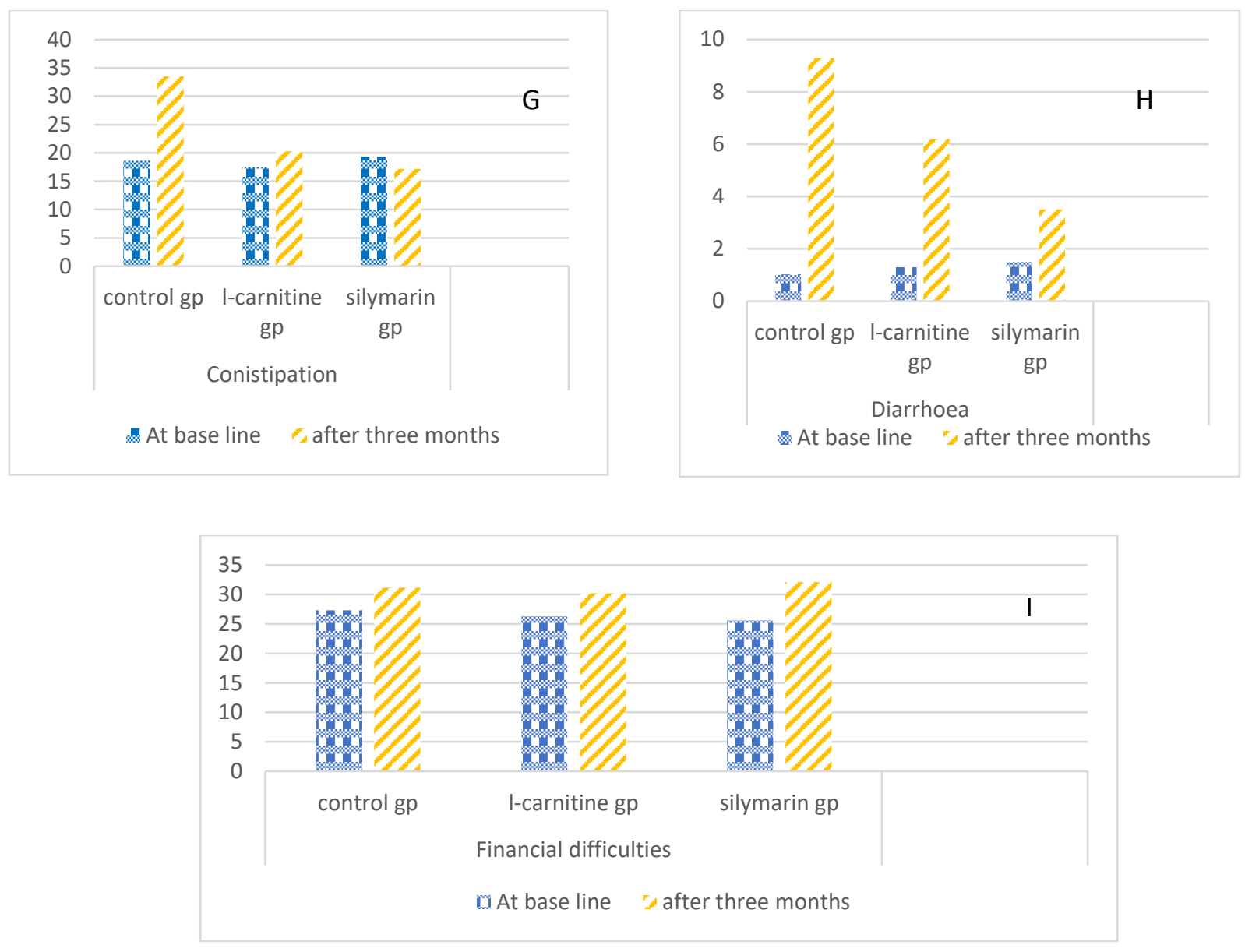

Figure (3): Comparison between studied groups regarding symptom scales- A: fatigue, B: nausea and vomiting, C: pain, D: dyspnoea, E: insomnia, F: appetite loss, G: constipation, H: diarrhoea,I: Financial difficulties (EORTC QLQC30) 

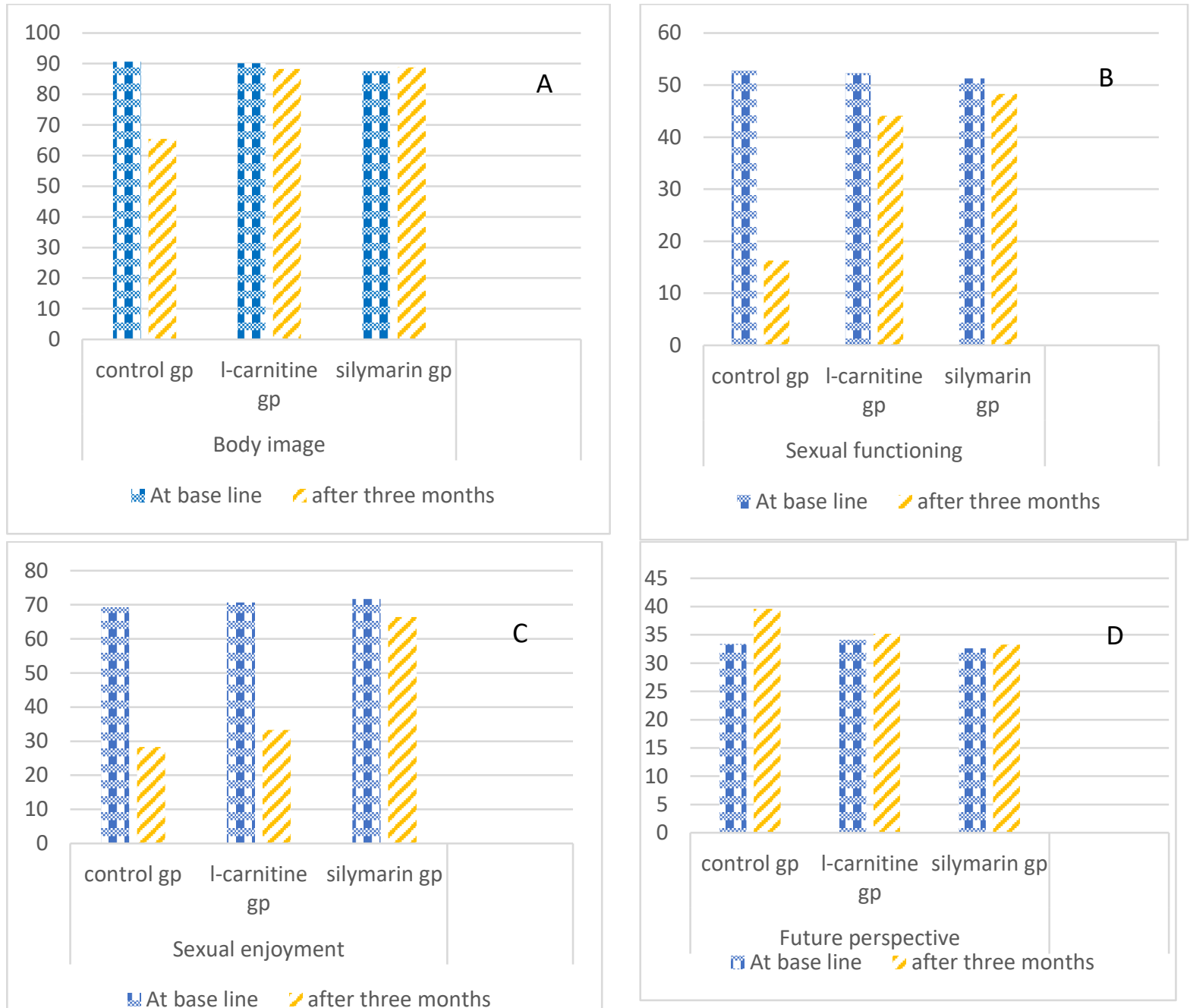

Figure (4): Comparison between studied groups regarding functional scales -A: body image, B: sexual functioning, C: sexual enjoyment, D: future perspective, (EORTC QLQ-BR23) 

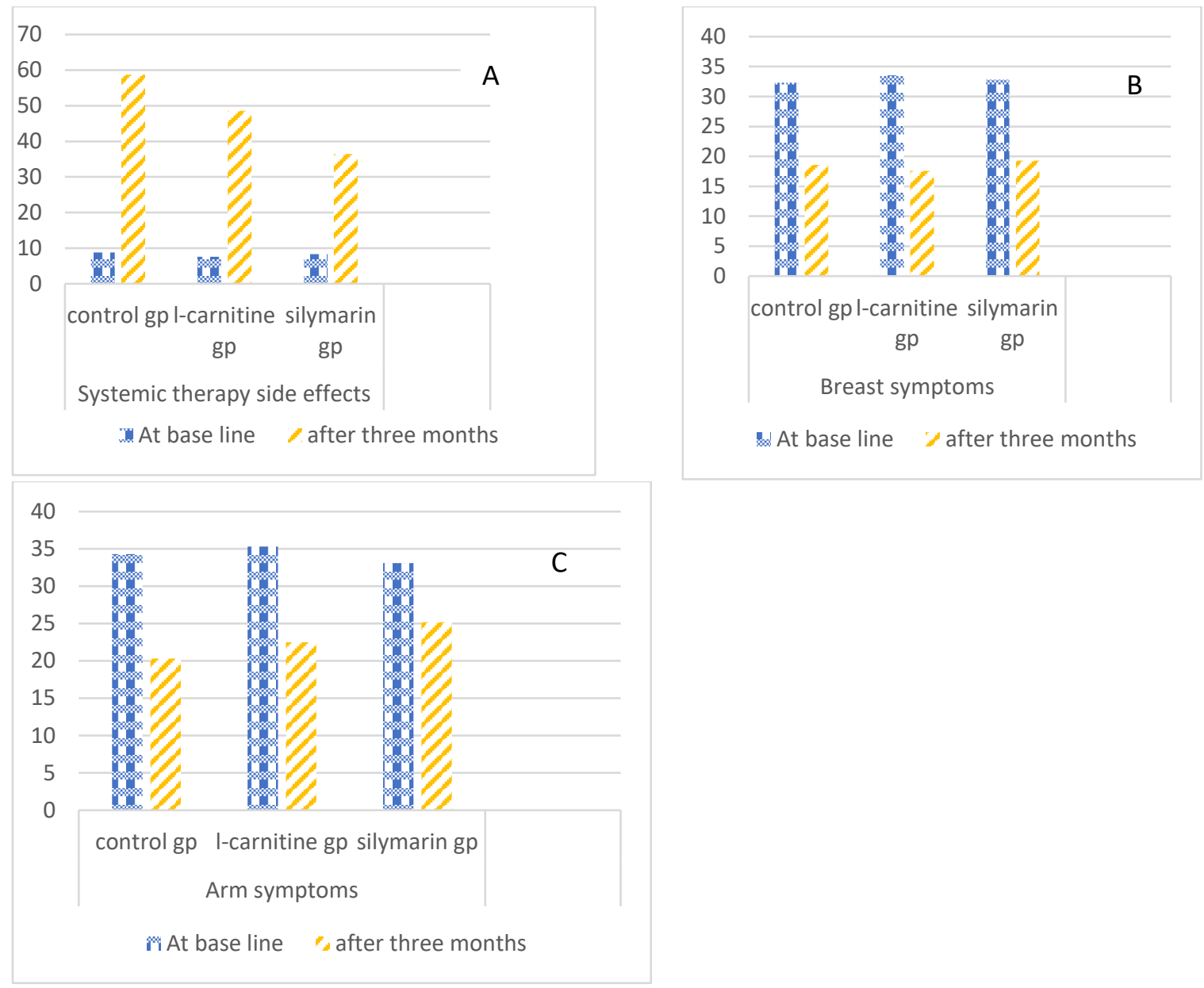

Figure (5): Comparison between studied groups regarding symptom scales - A: systemic therapy side effects, B: breast symptoms, C: arm symptoms (EORTC QLQ-BR23)

\section{Discussion}

The measurement of HRQoL is a valuable feature to determining the impact of medical care on diseases, psychological problems, lifetime happiness, and the well-being of patients. [15]. BC patients are at an increased risk for general HRQoL therapies (e.g., exhaustion, sleep problems, and pain) and psychological disorders (depression, anxiety, apprehension of recurrence, issues related to sex and body image) as well and general HRQoL. [16,17]. Chemotherapy often affects the patient's HRQoL expectations, as symptoms escalate and the functioning level declines. [1820]. It is necessary to add a new strategy to deal with chemotherapy's undesirable effects on patients' quality of life.

Our research is the first to examine the impact of silymarin and l-carnitine on the wellbeing of cancer patients undergoing anthracycline-based protocols and could also help oncologists assess classic symptoms that chemotherapy activates as well as the influence of chemotherapy, has psychosocial characteristics.

Our results showed that anthracycline has a negative effect on HRQoL, represented by a decline in global health status score, functional scales, and symptoms scale. This influence may be clarified by chemotherapy, which indicates that the 
health status of women with $\mathrm{BC}$ is worsened during therapy. Binnoto et al. stated that global health worsened also with the rising complications of chronic medication during chemotherapy therapy [21] In fact, BC patients have a high risk of developing behavioral changes that adversely affect HRQoL. [22]. According to previous studies $[23,24]$, the levels of psychological activity and body image during chemotherapy are substantially decreased, and modified body image is known to be a critical psychosocial issue for women with BC. [25]. This can be understood because the body image is influenced by the context of what others think and thus affects the trust of the individual. Psychological factors were strongly linked to global HRQoL and decreased social and emotional functioning in BC patients [26]. On comparing the results of the baseline scores and after 3 months, there was also a significant decline between the periods in cognitive functioning, pain, dyspnoea, and constipation between the two evaluations in the control group. These measures deal with physical effort, health, and support with basic needs and the ability to work or do daily work. This result is growing in patients with $\mathrm{BC}$ because of physical disabilities linked to illness and medication. The literature records similar results $[18,27]$. As the physical performance of the target is consistently better earlier than the end of the procedure, if exhaustion progresses [19]. Regarding the hair loss scale disturbance, segments cannot be contrasted. In line with the EORTC Rating Manual [14], a variance in the EORTC QLQ-BR23 answers is expected as measures disrupted by hair loss and sexual pleasure are not valid where the responses correlated with this measure are' no.' However, a high score on this examination suggests that the disorder adversely affected HRQoL.[14]. Alopecia is therefore life-altering and patients perceive these side effects as distressing [21]. This modification may create discomfort as to how others see it or evaluate it, causing social interaction to disappear because it feels uncomfortable in public places. [18]. The social isolation of $\mathrm{BC}$ patients is associated with a number of reasons. Social stigmatization of the disease can affect BC woman's interactions with other people. Our research has affected the social functioning of chemotherapy, indicating that a patient's physical condition and treatment interferes with family relations and social activities [21]. Similar results were reported in other studies [18], demonstrating the effect of chemotherapy on social relationships. On the other hand, larger social networks are linked to greater HRQoL when patients get better social support from family and friends after a BC diagnosis. [28]. It is known that attraction can be affected by shifts in hormone levels and changes in body image after a cancer diagnosis. [29]. Our findings are in agreement with the study of Hall et al [30]. which revealed that, in the short and long term, the majority of systemic effects of chemotherapy tend to affect women's sexuality. The findings of elevated systemic adverse effects (systemic medication side effects, exhaustion, nausea and vomiting, depression, loss of appetite, and diarrhea) in patients treated with chemotherapy are compatible with the expected results of toxicity for the drug. Chemotherapy may also worsen toxicity at low levels [31] such as diarrhea, which may be enough to worsen patients' HRQoL. In cancer patients, insomnia is also a common problem. Chemotherapy's concomitant effect on insomnia symptoms is mediated by various oncological symptoms, such as urinary symptoms, nausea, and night and digestive symptoms. [32]. Smell and taste alterations also take place as a side effect of chemotherapy. These changes affect food behavior, reduce food consumption, or limit food intake [33]. 
As illustrated in our results, the addition of 1carnitine to anthracycline-based chemotherapy protocol showed non-significant changes in the global health status scale score, functional scale scores, and symptom scale scores of insomnias, appetite loss, and diarrhea from baseline values. This may be due to that 1-carnitine (LC) plays an important role in the metabolism of fatty acids, and LC deficiency is associated with a feeling of weakness or general fatigue. Cancer patients receiving chemotherapy often develop l-carnitine deficiency, which is considered to be a factor contributing to general fatigue. [35]. There also was an improvement in body image score compared to the control group this explained as chemotherapy-induced damage of the carnitine system, and secondary deficiency of this molecule may cause fatigue due to impaired energy metabolism and thus bad impact on the selfconfidence of women with breast cancer [35]. Thus, restoration of the carnitine pool may alleviate the body image score of cancer patients. Our results are in harmony with Shindo et al. who studied the effect of 1-carnitine on the quality of life of cancer patients receiving chemotherapy and reported significant improvement from the control group [36].

MATSUI et al. also studied the impact of 1carnitine on the quality of life of cancer patients with chemotherapy reporting improvement [35].

These studies open the way for more research on the 1-carnitine impact on health-related quality of life of cancer patients, as it is a constrain of chemotherapy.

Regarding the administration of silymarin with anthracycline contains chemotherapy, our results showed that there was a non-significant change in global health status score, functional scale scores, and symptom scale scores including (fatigue, insomnia, appetite loss, and constipation) from the baseline. This may be interpreted as silymarin has been found to be a very potent antioxidant, supporting native cellular antioxidant mechanisms such as glutathione (GSH) and superoxide dismutase by scavenging free radicals and reactive oxygen species (ROS) [37]. This can partly explain the efficacy of silymarin in hepatic damage due to disease or poisons because this antioxidant action may reduce oxidative stress associated with lipid insults that suppress lipid peroxidation (and thus cell death). The general anticancer effects of flavonoids collectively as well as the high antioxidant ability of silymarin, there was a strong interest in modifying silymarin for use as a chemoprotective agent. [37].

Our study is the first to study the effect of silymarin on the health-related quality of life of cancer patients. There was also a non-significant

There also was an improvement in body image score compared to the control group, which may be due to the ability of silymarin to eliminate toxins that have undesirable effects on the mental status and self-pride sense of the patients.

More studies should be done in a similar population in order to analyzes the long-term HRQoL effects of silymarin and 1-carnitine on anthracycline-based chemotherapy

Physicians and healthcare professionals should often evaluate patients for side effects of preventive treatment and use symptom scales. Screening can also take into account patients ' views of global health status and QoL, physical functioning, job functioning, and emotional and social functioning. In this context, basic communication skills and sympathy in the psychosocial evaluation are significant. 
It is important to understand the patient and the family objectives in order to help the therapy tailor to its needs and to make ensure that we support the entire person, including cancer care and wellbeing preferences into consideration during therapy. Minimizing the adverse effects of medication and introducing strategies to help the patient overcome this process is critical for enhancing HRQoL. We must adapt our care strategy to the needs of each patient with this awareness.

\section{Conclusions}

In BC cases, the HRQoL of chemotherapy is usually worse during the third month relative to the time before therapy starts. The addition of 1carnitine and silymarin to anthracycline- based chemotherapy showed improvement in healthrelated quality of life of cancer patients and provided the basis for the design of future placebocontrolled supplementation studies in this population.

\section{Conflicts of interest}

The contributors cannot reveal conflicts of interest.

\section{Funding statement}

The writers provided no financial support for this article's study, authorship, and/or publishing.

All authors share the data underlying the findings of their manuscripts. Data sharing allows researchers to verify the results of an article, replicate the analysis, and conduct secondary analyses.

\section{References}

1. Jemal A, Vineis P, and Bray F, et al (2014) The Cancer Atlas 2nd edn (Atlanta: American Cancer Society) [www.cancer.org/canceratlas]

2. Ferlay J, Soerjomataram I, and Dikshit R, et al (2015) Cancer incidence and mortality worldwide: sources, methods and major patterns in GLOBOCAN 2012 Int J Cancer 136 E359-E386 https://doi.org/10.1002/ijc.29210

3. Anampa J, Makower D, and Sparano JA (2015) Progress in adjuvant chemotherapy for breast cancer: an overview BMC Med 13195 https://doi.org/10.1186/s12916-015-0439-8

PMID: 26278220 PMCID: 4538915

4. Chopra I and Kamal KM (2012) A systematic review of quality of life instruments in longterm breast cancer survivors Health Qual Life Outcomes 1014 https://doi.org/10.1186/14777525-10-14 PMID: 22289425 PMCID: 3280928

5. Ferreira RG and Franco LF de R (2017) Efeitos colaterais decorrentes do tratamento quimioterápico no câncer de mama: revisão bibliográfica Rev da Univ Val do Rio Verde https://doi.org/10.5892/ruvrd.v15i2.3759

6. Stanton AL, Rowland JH, and Ganz PA (2015) Life after diagnosis and treatment of cancer in adulthood: contributions from psychosocial oncology research Am Psychol 70 159-174 https://doi.org/10.1037/a0037875 PMID: 25730722

7. WHOQOL Group (1994) Development of the WHOQOL: rationale and current status Int $\mathbf{J}$ Ment Health 23 24-56 https://doi.org/10.1 $\underline{080 / 00207411.1994 .11449286}$

8. Food and Drug Administration (2006) Guidance for industry: patient-reported outcome measures: use in medical product development to support labeling claims: draft guidance Health Qual Life Outcomes 479 https://doi.org/10.1186/1477-7525-4-79 PMID: 17034633 PMCID: 1629006

9. Guyatt GH, Feeny DH, and Patrick DL (1993) Measuring health-related quality of life Ann 
Intern Med 118 622-629 https://doi. org/10.7326/0003-4819-118-8-19930415000009 PMID: 8452328

10. Sayed -Ahmed MM. Role of carnitine in cancer chemotherapy-induced multiple organ toxicity. Saudi pharmaceutical journal. October 2010;18(4) Pages 195-206

11. Surai, P.F. Silymarin as a Natural Antioxidant. An Overview of the Current Evidence and Perspectives. Antioxidants 2015, 4, 204-247.

12. Frassová Z, Rudá-Ku erová J. Milk Thistle (Silybum Marianum) as a supportive Phytotherapeutic agent in Oncology. Klin Onkol. 2017, 30(6), 426-432.

13. Zou, H.; Zhu, X.X .; Zhang, G.B.; Ma, Y.; Wu, Y.; Huang, D.S. Silibinin: an old drug for hematological disorders. Oncotarget $2017 \mathrm{Jul}$ 11; 8(51): 89307-89314.

14. Fayers P, Aaronson N, and Bjordal K, et al (2001) The EORTC QLQ-C30 Scoring Manual 3rd edn (Brussels: European Organisation for Research and Treatment of Cancer) [https://www.eortc.org/app/uploads/sites/2/201 8/02/SCmanual.pdf]

15. Montazeri A (2008) Health-related quality of life in breast cancer patients: a bibliographic review of the literature from 1974 to 2007 J Exp Clin Cancer $\quad$ Res $\quad 27 \quad 32$ https://doi.org/10.1186/1756-9966-27-32 PMID: 18759983 PMCID: 2543010

16. Fanakidou I, Zyga S, and Alikari V, et al (2018) Mental health, loneliness, and illness perception outcomes in quality of life among young breast cancer patients after mastectomy: the role of breast reconstruction Qual Life Res 27 539-543 https://doi.org/10.1007/s11136- 017-1735-x
17. Leinert E, Singer S, and Janni W, et al (2017) The impact of age on quality of life in breast cancer patients receiving adjuvant chemotherapy: a comparative analysis from the prospective multicenter randomized ADEBAR trial Clin Breast Cancer 17 100-106 https://doi.org/10.1016/j.clbc.2016.10.008

18. Gaton-Johansson F, Watkins CC, and Kanu IK, et al (2015) The effects of symptoms on quality of life during chemotherapy in africanamerican women with breast cancer J Natl Black Nurses Assoc 26 7-16

19. Tachi $\mathrm{T}$, Teramachi $\mathrm{H}$, and Tanaka $\mathrm{K}$, et al (2015) The impact of outpatient chemotherapyrelated adverse events on the quality of life of breast cancer patients PLoS One 10 e0124169 https:// doi.org/10.1371/journal.pone.0124169 PMID: 25915539 PMCID: 4410996

20. Binotto M, Reiner T, Werutsky G, Zaffaroni F and Schwartsmann G (2020) Health-related quality of life before and during chemotherapy in patients with early-stage breast cancer ecancer 2020, 14:1007; www.ecancer.org; DOI: https://doi.org/10.3332/ecancer.2020.1007

21. Barbosa PA, Cesca RG, and Pacífico TED, et al (2017) Quality of life in women with breast cancer, after surgical intervention, in a city in the zona da mata region in Minas Gerais, Brazil Rev Bras Saúde Matern Infant 17 385-399 https://doi.org/10.1590/180693042017000200010

22. Perroud HA, Alasino CM, and Rico MJ, et al (2016) Quality of life in patients with metastatic breast cancer treated with metronomic chemotherapy Futur Oncol 12 1233-1242 https://doi.org/10.2217/fon-2016-0075 
23. Ho SSM, So WKW, and Leung DYP, et al (2013) Anxiety, depression and quality of life in Chinese women with breast cancer during and after treatment: a comparative evaluation Eur $\mathbf{J}$ Oncol Nurs $17 \quad 877-882$ https://doi.org/10.1016/j.ejon.2013.04.005 PMID: 23727448

24. Pierrisnard C, Baciuchka M, and Mancini J, et al (2018) Body image and psychological distress in women with breast cancer: a French online survey on patients' perceptions and expectations Breast Cancer https://doi.org/10.1007/s12282-017-0828-2

25. Tang L, Fritzsche K, and Leonhart R, et al (2017) Emotional distress and dysfunctional illness perception are associated with low mental and physical quality of life in Chinese breast cancer patients Health Qual Life Outcomes $\quad 15 \quad 231$ https://doi.org/10.1186/s12955-017- 0803-9 PMID: 29191208 PMCID: 5709963

26. Zhang Y, Fritzsche K, and Leonhart R, et al (2014) Dysfunctional illness perception and illness behaviour associated with high somatic symptom severity and low quality of life in general hospital outpatients in China $\mathbf{J}$ Psychosom Res https://doi.org/10.1016/j.jpsychores.2014.06.00 5 PMID: 25149028

27. Winters ZE, Haviland J, and Balta V, et al (2013) Integration of patient-reported outcome measures with key clinical outcomes after immediate latissimus dorsi breast reconstruction and adjuvant treatment Br J Surg 100 240-251 https://doi.org/10.1002/bjs.8959

28. Kroenke CH, Kwan ML, and Neugut AI, et al (2013) Social networks, social support mechanisms, and quality of life after breast cancer diagnosis Breast Cancer Res Treat 139 515-527 https://doi.org/10.1007/s10549-0132477-2 PMID: 23657404 PMCID: 3906043

29. Lindau ST, Abramsohn EM, and Baron SR, et al (2016) Physical examination of the female cancer patient with sexual concerns: what oncologists and patients should expect from consultation with a specialist CA Cancer J Clin 66 241-263 https://doi.org/10.3322/ caac. 21337 PMID: 26784536 PMCID: 4860140

30. Hall E, Cameron D, and Waters R, et al (2014) Comparison of patient reported quality of life and impact of treatment side effects experienced with a taxane-containing regimen and standard anthracycline based chemotherapy for early breast cancer: 6year results from the UK TACT trial (CRUK/01/001) Eur J Cancer 50

2375-2389

https://doi.org/10.1016/j.ejca.2014.06.007

PMID: 25065293 PMCID: 4166460

31. Jolly TA, Williams GR, and Bushan S, et al (2016) Adjuvant treatment for older women with invasive breast cancer Women's Heal 12 129-145 quiz 145-146

32. Savard J, Ivers H, and Savard M-H, et al (2015) Cancer treatments and their side effects are associated with aggravation of insomnia: results of a longitudinal study Cancer 121 1703-1711 https://doi.org/10.1002/cncr.29244 PMID: 25677509

33. Laviano A, Koverech A, and Seelaender M (2017) Assessing pathophysiology of cancer anorexia Curr Opin Clin Nutr Metab Care 20 340-345 https://doi.org/10.1097/MCO.00000000000003 94 PMID: 28598896 
34. Velikova G, Booth L, and Smith AB, et al (2004) Measuring quality of life in routine oncology practice improves communication and patient well-being: a randomized controlled trial J Clin Oncol $22 \quad 714-724$ https://doi.org/10.1200/JCO.2004.06.078

35. MATSUI1 H, EINAMA1 T, SHICHI1 S, et al (2018) L-Carnitine supplementation reduces the general fatigue of cancer patients during chemotherapy MOLECULAR AND CLINICAL ONCOLOGY 8: 413-416
36. Shindo1 T \& Kobayashi K \& Tanaka $\mathrm{T} \&$ Masumori N et al (2019) Can levocarnitine supplementation improve fatigue caused by sunitinib as a treatment for renal cell carcinoma? A single-center prospective pilot study Supportive Care in Cancer 27:1491-1496 https://doi.org/10.1007/s00520-018-4521-6

37. Ting H, Deep G, and Agarwal R (2013) Molecular Mechanisms of Silibinin-Mediated Cancer Chemoprevention with Major Emphasis on Prostate Cancer The AAPS Journal, Vol. 15, No. 3,707-716 